\title{
EXTRACTION OF UNIFORMLY ACCURATE PHASE FUNCTIONS ACROSS SMOOTH SHADOW BOUNDARIES IN HIGH FREQUENCY SCATTERING PROBLEMS*
}

\author{
ANDREAS ASHEIM ${ }^{\dagger}$ AND DAAN HUYBRECHS $\ddagger$
}

\begin{abstract}
Several recent numerical schemes for high frequency scattering simulations are based on the extraction of known phase functions from an oscillatory solution. The remaining function is typically no longer oscillatory, and as such it can be approximated numerically with a number of degrees of freedom that does not depend on the frequency of the original problem. Knowledge of the phase of a solution typically comes from asymptotic analysis, for example, from geometrical optics. We consider integral equation formulations of time-harmonic scattering by a smooth and convex obstacle and focus on the so-called shadow boundaries. They are the points where the incoming waves are tangential to the boundary of the scatterer. We devise a numerical method that incorporates advanced results from asymptotic analysis which describe the frequency-dependent transitional behavior of the solution uniformly across these points. We describe and resolve an apparent conflict between two theories that describe the asymptotic behavior of this problem. They are the well-known geometric theory of diffraction and the rigorous asymptotic analysis by Melrose and Taylor, based on microlocal analysis.
\end{abstract}

Key words. scattering problems, high frequency, asymptotics

AMS subject classifications. Primary, 65N38; Secondary, 45B05, 34E05

DOI. $10.1137 / 130936075$

1. Introduction. This work concentrates on the model problem of time-harmonic scattering as described by the Helmholtz equation,

$$
\Delta u+k^{2} u=0,
$$

where $k$ is the wavenumber, which can be large. An incident wave is scattered by an obstacle $\Omega$, and we are interested in computing the scattered field. More specifically, we consider the problem of computing the scattered field when the wavenumber $k$ is large or moderately large.

Large $k$ asymptotic methods for wave scattering problems have a long history. The simplest technique is arguably the geometrical optics (GO) approximation, where the field is represented conceptually in terms of propagating rays. Although immensely useful in many cases, the GO approximation has several limitations. First, it does not account for the nonzero field in the shadow of a scatterer, as well as close to the shadow boundary. Moreover, the approximation is not valid near caustics, i.e., points where the field is determined by more than one ray. More specialized asymptotics take into account the deflection of rays into the shadow, e.g., as in Keller's geometric theory of diffraction (GTD) [19]. Other examples include, among others, uniform asymptotic expansions for transition regions [22] and uniform expansions valid across

* Received by the editors September 9, 2013; accepted for publication (in revised form) December 26, 2013; published electronically April 10, 2014. This work was supported by the Norwegian Research Council's Magne S. Espedahl fellowship.

http://www.siam.org/journals/siap/74-2/93607.html

†DAMTP, University of Cambridge, Wilberforce Road, CB3 0WA, Cambridge, UK (A.Asheim@ damtp.cam.ac.uk).

†Departement Computerwetenschappen, KU Leuven, 3001 Heverlee, Belgium (daan.huybrechs@ cs.kuleuven.be). 
shadow boundaries [2, 27], near edges [21], and caustics [23]. Being asymptotic approximations, such methods are of limited use in low wavenumber problems.

Discretization methods for scattering problems have developed with relatively little input from asymptotics. Finite element and finite difference methods (FEM/FD) for scattering problems are based on discretizing partial differential equations (PDEs), such as (1.1), and solving the resulting systems of equations. Because of sampling requirements, such methods have a computational complexity that grows at least as fast as $\mathcal{O}\left(k^{d}\right)$, where $d$ is the dimesion of the problem. Boundary element methods (BEM) are based on solving integral equations on the boundary of $\Omega$ and can therefore do with $\mathcal{O}\left(k^{d-1}\right)$ degrees of freedom, at the cost of having dense discretization matrices. For large enough wavenumbers, such methods are clearly still unsuited.

Hybrid methods that combine properties of asymptotic techniques and discretization methods have been proposed in order to efficiently treat moderate to high wavenumber problems. A common technique for such methods is phase extraction: the unknown is written as the product of a known phase function, usually obtained from the GO approximation, and an unknown amplitude function which is assumed to be less oscillatory than the original unknown. The feasibility of this technique has been demonstrated for smooth and convex two-dimensional scatterers $[8,4,15]$, as well as for smooth and convex three-dimensional scatterers $[3,11]$. The less oscillatory field implies that fewer degrees of freedom are needed for representing it. Such methods result in dense discretization matrices whose entries are oscillatory integrals. Efficient techniques for handling such integrals are available, and examples include Filon-type methods [17, 9] and numerical steepest descent methods [14]. In [15] it is shown that a sparse discretization can be obtained by approximating the oscillatory integrals with a Filon-type method. This approximation renders the dense operator representation sparse, with dense submatrices for the discretization of shadow- and transitional regions only, thereby removing one of the drawbacks of BEM methods. Several high-frequency techniques are comprehensively reviewed in [6].

These hybrid methods are based on using a priori knowledge about the solution, i.e., its asymptotic behavior, in order to make numerical solvers more efficient. From this perspective a natural question is how much such knowledge one can include and to what extent the added complexity of the solver results in improved efficiency of the computations.

Giladi [12] presents experiments extracting the phase function obtained from GTD in the shadow of the scatterer, thus further reducing the number of unknowns since the field in the shadow is less oscillatory compared to extracting the GO phase. If one wants to factor out more adverse wavenumber dependence, one has to properly treat the transition between the illuminated and shadow region as well. This transition can be described using uniform asymptotics. In this work we explore numerical methods that factor out essentially all adverse wavenumber dependence in such cases, and we aim to quantify the maximal gain to be had from such an approach. The ingredients of our methods come from the classical works on uniform asymptotics. The field of uniform asymptotics for scattering problems was a field of intense studies in the 1960s and 1970s and has since been viewed as mature. Yet, these results have, to the best of our knowledge, seen little use in computations, though they do appear in the analysis of numerical schemes (see, e.g., [8]).

The exposition continues with a statement of the problem in section 2 , followed by a review of the different asymptotic regions of the surface of a smooth, convex scatterer in section 3. This review leads to a discussion on the validity of GTD and uniform asymptotics as described by Melrose and Taylor [27] in the shadow side of a 
scatterer. We observe a disagreement between these two approximations which turns out to be rather relevant for numerical methods. Finally, several ways of exploiting this knowledge in numerical methods are presented in section 4 , and numerical experiments demonstrating the efficiency of the methods in section 5 .

\section{Problem statement.}

2.1. Integral equation formulation. Consider the scattering of time harmonic waves by a two-dimensional bounded scatterer $\Omega \in \mathbb{R}^{2}$ with a smooth boundary $\Gamma=\partial \Omega$. The total field outside the obstacle can be written as the sum of an incident wave $u^{i}$ and a scattered wave $u^{s}$, which solves the two-dimensional Helmholtz equation in the exterior space:

$$
\begin{aligned}
\Delta u^{s}+k^{2} u^{s} & =0, \quad x \in \mathbb{R}^{2} \backslash \bar{\Omega}, \\
u^{s}(x) & =-u^{i}(x), \quad x \in \Gamma .
\end{aligned}
$$

The Dirichlet boundary condition implies that the total field $u^{i}+u^{s}$ vanishes on $\Gamma$, corresponding to a perfectly reflecting object. The incoming wave $u^{i}$, which solves the Helmholtz equation as well, is assumed to be known. We further impose the Sommerfeld radiation condition on $u^{s}$ in order to ensure uniqueness of the solution [7].

The unknown scattered wave can be expressed using the single-layer potential

$$
u^{s}(x)=(S q)(x)=\int_{\Gamma} G(x, y) q(y) \mathrm{d} s_{y} .
$$

Here, $q$ is the single-layer potential density and

$$
G(x, y)=\frac{i}{4} H_{0}^{(1)}(k|x-y|)
$$

is the Green's function of the two-dimensional Helmholtz equation. Through jump relations it is shown that the density function can be found from the integral equation of the first kind [29]

$$
\int_{\Gamma} G(x, y) q(y) \mathrm{d} s_{y}=-u^{i}(x), \quad x \in \Gamma .
$$

It should be noted that other integral formulations are needed to guarantee solvability of the integral equation for all values of the wavenumbers [7]. However, for the purposes of this paper, (2.3) is sufficient.

In the following, the discussion is limited to incident plane waves,

$$
u^{i}(x)=\mathrm{e}^{\mathrm{i} k d \cdot x},
$$

and we shall only consider the integral equation formulation (2.3). Moreover, the scatterer $\Omega$ is assumed to be convex. Similar convexity conditions are assumed throughout the literature [6]. In an asymptotic setting, it may be possible to relax the convexity condition to a much weaker condition of local convexity [1], but that approach is not pursued further within this paper.

2.2. Parametrization. We assume a natural (arc-length) parametrization $\gamma$ of the boundary of the scatterer $\Omega$ is given,

$$
\gamma:[0, L] \rightarrow \partial \Omega
$$



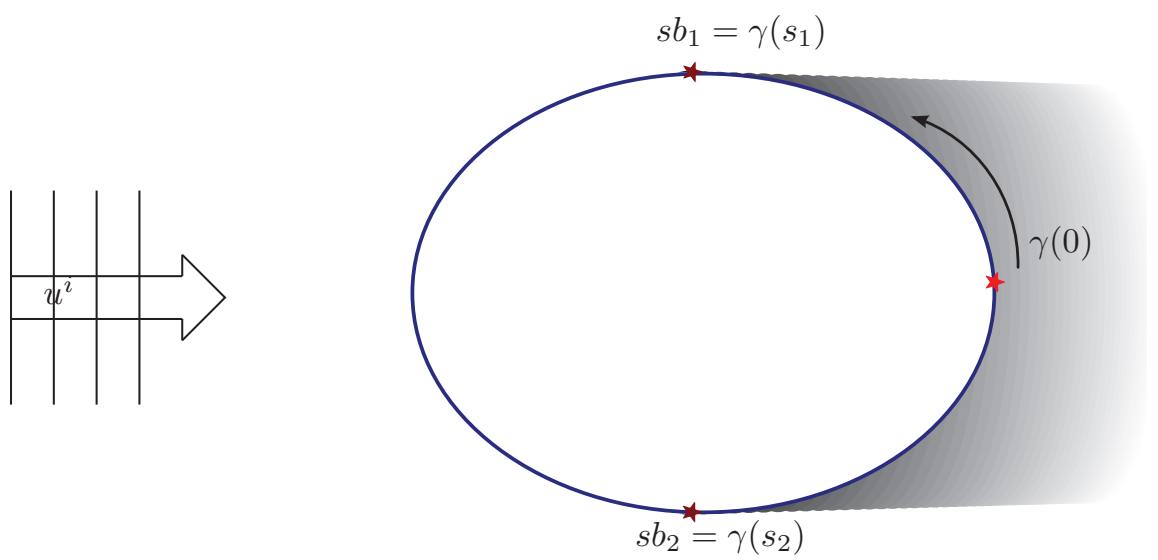

FIG. 1. Natural parametrization of the boundary, with $s_{1}$ and $s_{2}$ defined relative to the orientation of the parametrization.

Here, $L$ is the circumference. Using the natural parametrization in our approach serves merely to simplify the computation of the phase in the shadow region later on, at the cost of having to compute this parametrization. Any other parametrization can be used too, at the cost of having to do more computations for the phase later on.

Rewriting integral equation (2.3) using this parametrization yields

$$
\int_{0}^{L} K(\tau, t) q(\tau) \mathrm{d} \tau=-e^{i k d \cdot \gamma(t)}, \quad t \in[0, L],
$$

where $K(\tau, t)=G(\gamma(\tau), \gamma(t))$ and $q(t)$ denotes, in a slight abuse of notation, $q(\gamma(t))$. Similarly, in the following, the notation $u(t)$ is used to denote the field evaluated at the scatterer at the point $\gamma(t) \in \partial \Omega$.

For each convex obstacle with an incident plane wave, there are two shadow boundary points. They are denoted by $s b_{1}=\gamma\left(s_{1}\right)$ and $s b_{2}=\gamma\left(s_{2}\right)$ with $s_{1}, s_{2} \in[0, L]$ the corresponding points in the parameter domain. Without loss of generality, we assume that $\gamma(0)$ corresponds to the midpoint of the shadow region, i.e., $L-s_{2}=s_{1}$, and that the parametrization is oriented such that $s_{1}<s_{2}$, as illustrated in Figure 1 . Whenever we wish to refer to a shadow boundary without specifying which one, we shall denote this by $s$. Finally, $\kappa$ denotes the curvature of the obstacle, $\kappa(t)=\left\|\gamma^{\prime \prime}(t)\right\|$.

2.3. Phase extraction and high frequency formulations. As the wavenumber $k$ increases, the single-layer potential density $q$ becomes increasingly oscillatory. A classical discretization of (2.1) would therefore require an increasing number of discretization points. However, the nature of the oscillations is known from high frequency asymptotics for the scattering problem. For example, the Kirchhoff approximation [27], which is valid in illuminated regions of convex obstacles, predicts that

$$
q(t) \sim-2 \mathrm{i} k\left(d \cdot n_{\gamma(t)}\right) \mathrm{e}^{\mathrm{i} k d \cdot \gamma(t)}, \quad k \gg 0,
$$

where $n_{y}$ is the surface normal of $\Gamma$ at the point $y$. The amplitude of this approximation is nonoscillatory, and one may reasonably assume that the exact solution has the same form. Therefore, reformulating the scattering problem so that one solves for 
this amplitude means that a smaller number of degrees of freedom is needed, at least in the illuminated region. More generally, if a function $\Phi(t)$ contains the oscillations of $q(t)$ on $\partial \Omega$, such as $\Phi(t)=e^{i k d \cdot \gamma(t)}$ above, we can define a "smooth" solution $q_{s}(t)$ by

$$
q(t)=q_{s}(t) \Phi(t)
$$

such that a coarser discretization can be used for $q_{s}(t)$. Thus, we solve the integral equation

$$
\int_{0}^{L} K(\tau, t) q_{s}(\tau) \Phi(\tau) \mathrm{d} \tau=-e^{i k d \cdot \gamma(t)}, \quad t \in[0, L]
$$

for the unknown $q_{s}(\tau)$. In the following, several functions $\Phi$ are constructed with the property that they contain information about the solution, and they are referred to as phase functions.

Note, however, that the entries of the discretization matrix now consist of oscillatory integrals. Typically, a collocation method results in entries of the form

$$
\int_{\Gamma} K\left(t_{i}, \tau\right) \Phi(\tau) \phi_{j}(\tau) \mathrm{d} \tau
$$

where $t_{i}$ and $\phi_{j}$ are collocation points and basis functions, respectively. Quadrature methods are not discussed in this paper, but it is implied that efficient schemes for resulting oscillatory integrals can be devised. We refer the reader to [13] for a survey of such schemes and to [15] for a specific implementation in the context of a highfrequency scattering problem.

3. Asymptotics of scattering by a smooth and convex obstacle. In this section a brief review of the asymptotics of the smooth, convex problem is presented. We shall primarily discuss the behavior of the field on the surface of the scatterer, which is exactly what is needed for phase extraction. On the surface of the obstacle we distinguish three asymptotic regimes:

- the illuminated region,

- the shadow region, and

- two transitional regions (also known as the Fock regions).

They are illustrated in Figure 2. A review of the governing asymptotics in these regions, as well as in several other regions in the field around the obstacle, is found in [30]. This review is in turn based on classical works [19, 2].

An apparent contradiction is observed between GTD and the theory of Melrose and Taylor, namely, a discrepancy in the phase of the solution in the shadow region. A new phase function is constructed by combining both theories, and this is shown to work well in practice. Numerical experiments are presented in this section that demonstrate the behavior of the solution $q(t)$ of the scattering problem after extraction of various proposed phase functions.

3.1. Illuminated region: GO. The asymptotics for the illuminated side of a convex problem is described by GO. The first term of the GO approximation is obtained by using an ansatz for the solution of the Helmholtz equation of the form

$$
u^{s}(x) \sim a(x) e^{\mathrm{i} k \phi(x)}, \quad k \rightarrow \infty .
$$




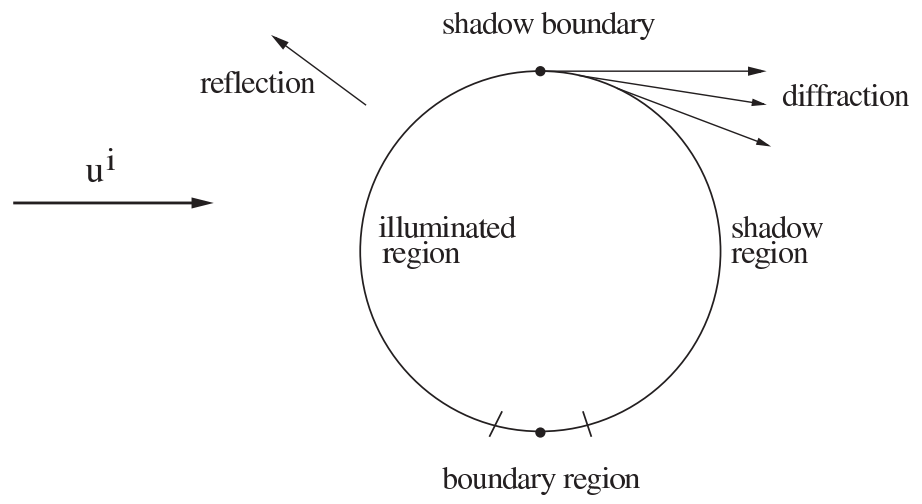

FIG. 2. Three asymptotic regions.

Solving the Helmholtz equation to lowest order yields the eikonal and transport equations for the phase and amplitude functions,

$$
|\nabla \phi|=1, \quad 2 \nabla \phi \cdot \nabla a+\Delta \phi a=0 .
$$

The eikonal equation defines the rays. It can be solved, e.g., by the method of characteristics with initial conditions given by the boundary condition [2]. In homogenous media, these rays are straight lines and they do not penetrate into the shadowed field, behind the obstacle. GO therefore does not account for the nonzero field in the shadow of the obstacle.

In our setting, GO predicts the phase of the single layer density along the scatterer to be precisely that of the incoming wave, as in the Kirchhoff approximation (2.6). Thus, for the incoming plane wave (2.4), the solution behaves as

$$
u(t) \sim c(t) e^{\mathrm{i} k d \cdot \gamma(t)}, \quad k \rightarrow \infty, \quad t \in(s 1, s 2),
$$

where $c(t)$ is a smooth and wavenumber-independent function (in the sense that $c(t)$ and its derivatives are bounded with respect to growing $k$ ). A common approach to exploit this asymptotic property is by factoring out the phase function $[4,8,14,11]$

$$
\Phi^{G O}(t)=e^{\mathrm{i} k d \cdot \gamma(t)} .
$$

If one factors out a different function near the shadow boundary, then it should reduce to a smooth multiple of $\Phi^{G O}(t)$ in the illuminated region in order to maintain a nonoscillatory solution in this region.

3.2. The shadow region: GTD. The GTD approximation due to Keller [19] uses Fermat's principle to extend the GO solution into the shadow: rays are such that their optical path length is stationary. A ray can move into the shadow by striking the obstacle tangentially at a shadow boundary point $s$, moving along the boundary for a while, and then exiting tangentially as shown in Figure 3. The ray along the boundary is commonly called a creeping ray. An expression for the creeping ray involves the local curvature $\kappa[18]$ :

$$
u(t) \sim c(t) e^{i k|t-s|} e^{i \alpha_{0} k^{1 / 3} \int_{s}^{t} \kappa^{2 / 3}(\tau) \mathrm{d} \tau}, \quad k \rightarrow \infty,
$$

for $t$ in the shadow region and where $c(t)$ is again a smooth and wavenumber independent function. This expression was derived for any $t$ that is a fixed distance $\mathcal{O}(1)$ away from $s$, which implies in particular that $t$ may not coincide with $s$. 


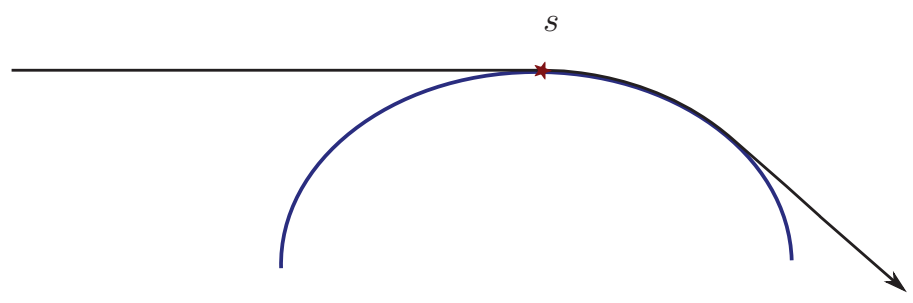

FIG. 3. A GTD ray.

Keller notes that (3.2) is only the first term in a countable series over the modes $\alpha_{n}$. In [18] the expression

$$
\alpha_{n} \approx \frac{1}{2}\left[3 \pi\left(n+\frac{3}{4}\right)\right]^{2 / 3} e^{i \pi / 3}
$$

is used. A more accurate expression is

$$
\alpha_{n}=\nu_{n+1} 2^{-1 / 3} e^{-2 \pi i / 3},
$$

where $\nu_{n}$ is the $n$th root of the Airy function. The former appears from the latter by considering the large $n$ asymptotics of the roots of the Airy function. Note that rays can also travel around the obstacles indefinitely, obtained by replacing $t$ in (3.2) by $t+m L, m \in \mathbb{N}$. Finally, there are two series in total, one for each shadow boundary point, travelling around the obstacle in opposite directions [18].

The imaginary part of the exponent in the factor $\exp \left(i \alpha_{0} k^{1 / 3} \int_{s}^{s+t} \kappa^{2 / 3}(\tau) \mathrm{d} \tau\right)$ results in exponential decay of the solution in the shadow. This accounts for the attenuation of the wave due to the shedding of energy in the tangential direction. It is obtained under the assumption that this shedding only depends on the boundary conditions and on geometric properties of the obstacle.

The dominant part of the phase in expression (3.2) is the factor $|t-s|$. This is precisely the arclength of the trajectory along $\partial \Omega$ connecting the point $\gamma(t)$ to the shadow boundary point at $\gamma(s)$. For the purpose of phase extraction in the shadow region, one may define the phase function

$$
\Phi^{G T D}(t)=e^{i k|t-s|} e^{i \alpha_{0} k^{1 / 3} \int_{s}^{t} \kappa^{2 / 3}(\tau) \mathrm{d} \tau} .
$$

This phase function is suitable for extraction in the shadow region near the shadow boundary $s$, as long as $t$ remains bounded away from $s$ for increasing frequency. For convex obstacles in two dimensions, the validity of this phase function is also restricted by the creeping ray coming from the other shadow boundary: a different approach is called for in the region where both creeping rays are of similar size.

If one factors out a different function near the shadow boundary, then it should reduce to a smooth multiple of $\Phi^{G T D}(t)$ in the shadow region in order to maintain a nonoscillatory solution in this region.

3.3. Transitional region. The transitional behavior of the solution near the shadow boundary, from the illuminated region into the shadow, has been extensively 
studied. The developments started with the work of Fock [10], resulting in the name Fock domain for the transitional region. Contributions were made by Ludwig [24], Morawetz and Ludwig [28], Babich and Buldyrev [2], Buslaev [5], and others. We refer to [2, section 13.9] and [8, section 5] for a detailed account of the history with references. In this section we focus on the uniform expansion of Melrose and Taylor [27], because the formulation is particularly convenient to use for phase extraction. The Melrose-Taylor expansion is valid in a region across the shadow boundary that does not depend on the wavenumber $k$ and thus seems well suited to connect the illuminated and shadow regions in a wavenumber-independent way.

It should be noted that most uniform expressions in literature which are explicit in the parameters of the problem, in the sense that they do not require the solution of a problem-dependent PDE or ODE, are valid only in a shrinking region of size $\mathcal{O}\left(k^{-1 / 3}\right)$ around the shadow boundary (e.g., [2, section 13.7], [30, section 2.2]). A further approximation often made in practice is to assume that the curvature of the obstacle is constant near the shadow boundary. We elaborate briefly on the nature of the approximation that results in the limitation on the validity of uniform expansions. Next, we formulate the Melrose-Taylor expansion. Though this expansion is valid mathematically in a region that does not depend on the wavenumber $k$, it is not unaffected by the approximation in practice.

3.3.1. The Fock-Leontovic equation. In the transitional region near the shadow boundary, neither GTD nor GO apply. The nature of the scattering problem is described by the Fock-Leontovic equation. Here, we briefly summarize its derivation based on the description in the review [30].

We may use the following ansatz for the Helmholtz equation

$$
u^{s}(t, n) \sim A(t, n) e^{i k t},
$$

where $t$ refers to position along the scatterer and $n$ is the normal distance to the scatterer. Investigating $A(t, n)$ in the vicinity of a shadow boundary leads to an appropriate rescaling of variables,

$$
t=s+k^{-1 / 3} \kappa(s)^{-2 / 3} \hat{t}
$$

and

$$
n=k^{-2 / 3} \kappa(s)^{-1 / 3} \hat{n} .
$$

With this rescaling, the leading order term of $\hat{A}(\hat{t}, \hat{n})=A(t, n)$ solves the FockLeontovič equation,

$$
\frac{\partial^{2} \hat{A}}{\partial \hat{n}^{2}}+2 i \frac{\partial \hat{A}}{\partial \hat{t}}+2 \hat{n} \hat{A}=0
$$

with approximate boundary condition

$$
\hat{A}(\hat{t}, 0)=-e^{-i \hat{t}^{3} / 6} .
$$

This boundary condition ensures that the actual Dirichlet boundary condition is satisfied to third order close to the shadow boundary. The amplitude $\hat{A}$ can be obtained from a Fourier transform of (3.6),

$$
\hat{A}(\hat{t}, \hat{n})=-2^{1 / 3} \int_{-\infty}^{\infty} \frac{\operatorname{Ai}\left(-2^{1 / 3} z\right) \operatorname{Ai}\left(-2^{1 / 3}(z+\hat{n}) e^{2 \pi i / 3}\right)}{\operatorname{Ai}\left(-2^{1 / 3} e^{2 \pi i / 3} z\right)} e^{-i \hat{t} z} \mathrm{~d} z .
$$


The value of the single layer density $q(t)$ is found by taking the normal derivative of this expression at $n=0$, i.e., on the surface of the scatterer.

We make two remarks:

- The rescaling (3.5) is a natural one in this setting and demonstrates that the transition from illuminated to shadow regions occurs in a region of size $\mathcal{O}\left(k^{-1 / 3}\right)$.

- The approximate nature of the boundary condition (3.7) limits the accuracy of (3.8) outside of this region.

3.3.2. The uniform asymptotics of Melrose and Taylor. Three attractive features of the Melrose-Taylor (hereafter abbreviated MT) expansion for the purpose of numerical simulations are the following:

- The influence of the geometric properties of the obstacle is confined to one single function $Z(t)$, for which an explicit expression is available.

- The uniform character of the expansion is captured by a single special function, $\Psi(\tau)$, which can be approximated a priori, regardless of the shape of the obstacle.

- The expansion is valid in a region across the shadow boundary that does not depend on the wavenumber.

Though both functions $Z$ and $\Psi$ can be found earlier in the literature; this uniform expansion was independently rigorously proved using microlocal analysis in [27]. Several clarifications of the formulas are given in [8, section 5]. Here, we formulate the result in essentially the same form as in [8, Theorem 5.1].

THEOREM 1. There exists $\Delta>0$ such that

$$
q(t) \sim e^{i k d \cdot \gamma(t)} \sum_{l, m \geq 0} k^{2 / 3-2 l / 3-m} b_{l, m}(t) \Psi^{(l)}\left(k^{1 / 3} Z(t)\right),
$$

valid for $t \in I_{\Delta}:=\left[s_{1}-\Delta, s_{1}+\Delta\right] \cup\left[s_{2}-\Delta, s_{2}+\Delta\right]$. The functions $b_{l, m}, \Psi$ and $Z$ have the following properties:

1. $b_{l, m}(t)$ are complex-valued $C^{\infty}$ functions on $I_{\Delta}$.

2. $Z(t)$ is a $C^{\infty}$ and real-valued function on $I_{\Delta}$ with simple zeros at $t=s_{1}$ and $t=s_{2}$, being positive for $\left(s_{1}, s_{2}\right) \cap I_{\Delta}$ and negative otherwise.

3. $\Psi$ is an entire function specified by

$$
\Psi(\tau)=e^{-i \frac{\tau^{3}}{3}} \int_{\Gamma} \frac{e^{i z \tau}}{A i\left(e^{2 \pi i / 3} z\right)} \mathrm{d} z,
$$

where $A i$ is the Airy function and $\Gamma$ is a path that connects $\infty e^{i(\pi-\delta)}$ with $+\infty$ for $\delta>0$ sufficiently small.

The function $\Psi(\tau)$ is also called the Fock function, with its integral representation referred to as the Fock integral [10]. For the precise meaning of the asymptotic expansion one employs the symbolic classes of Hörmander, as detailed in [27]. Essentially, the difference between the left-hand side and a number of terms of the right-hand side decays at a certain algebraic rate in $k$.

In the following we are only concerned with the first term in this expansion. The phase function that presents itself is the leading order term of (3.9),

$$
\Phi^{M T}(t)=e^{i k d \cdot \gamma(t)} b_{0,0}(t) \Psi\left(k^{1 / 3} Z(t)\right) .
$$

This function should be a smooth multiple of the GO phase function (3.1) in the illuminated region and of the GTD phase function (3.4) in the shadow region. We intend to verify both. 
First, the function $b_{0,0}(t)$ is determined by requiring that the expansion (3.9) agrees with the Kirchhoff approximation in the illuminated region. Since the function $Z(t)$ is positive on the illuminated side, the MT expansion reduces to the Kirchhoff approximation through the asymptotic behavior of $\Psi(\tau)$ for large and positive $\tau$. Asymptotically, it is known that

$$
\Psi(\tau) \sim-2 i \tau, \quad \tau \rightarrow \infty,
$$

and we must, by (2.6), have,

$$
b_{0,0}(t)=\frac{n(t) \cdot d}{Z(t)}
$$

The reduction to the Kirchhoff approximation is given with more detail in [8] and [27]. In particular, all terms in the expansion of $\Psi(\tau)$ for $\tau \rightarrow+\infty$ can be obtained.

It is instructive to also study how the MT expansion reduces to the asymptotics in the shadow region away from the shadow boundary. In spite of the uniform character of the MT expansion, surprisingly it does not accurately describe the creeping rays.

3.3.3. MT expansion versus GTD in the deep shadow. We consider the behavior of the MT expansion at a point $t$ in the shadow region that is a bounded distance away from the shadow boundary - a regime commonly called the deep shadow [2, section 13.8], [30, section 2.2]. This follows from the behavior of $\Psi(\tau)$ for large negative arguments. Using residue calculus, one finds that

$$
\Psi(\tau) \sim e^{-i \tau^{3} / 3-i \tau e^{-2 \pi i / 3} \nu_{1}}\left(1+\mathcal{O}\left(e^{-|\tau| \beta}\right)\right), \quad \tau \rightarrow-\infty,
$$

where $\nu_{1}=-2.33811 \ldots$ is the smallest root (in absolute value) of the Airy function.

The expansion of $\Psi$ can be used to deduce an expression for $Z(t)$. The wavenumberdependent components of the MT expansion are confined to the terms

$$
e^{i k d \cdot \gamma(t)} \Psi\left(k^{1 / 3} Z(t)\right),
$$

which in view of (3.12) reduce for negative $Z(t)$ asymptotically to

$$
e^{i k d \cdot \gamma(t)} e^{-i k Z(t)^{3} / 3-i k^{1 / 3} Z(t) e^{-2 \pi i / 3} \nu_{1}} .
$$

Identifying the $k^{1 / 3}$ with the corresponding term in (3.2), and using expression (3.3) for the modes $\alpha_{0}$, the appropriate form of the function $Z$ in neighborhoods around each of the shadow boundaries $s_{1}$ and $s_{2}$ is, respectively,

$$
\begin{aligned}
& Z_{1}(t)=2^{-1 / 3} \int_{s_{1}}^{t} \kappa^{2 / 3}(\tau) \mathrm{d} \tau \\
& Z_{2}(t)=-2^{-1 / 3} \int_{s_{2}}^{t} \kappa^{2 / 3}(\tau) \mathrm{d} \tau .
\end{aligned}
$$

However, the identification of (3.13) with the GTD expression (3.2) is not complete. Let us fix the shadow bounday point $s=s_{1}$. The expressions for $s_{2}$ are similar. The leading order phase term of (3.13) is

$$
k d \cdot \gamma(t)-k Z_{1}(t)^{3} / 3
$$


while the leading order phase term of GTD is

$$
\left|t-s_{1}\right|=s_{1}-t
$$

We expand the former around the point $s_{1}$. By construction we have that $d \cdot \gamma^{\prime}\left(s_{1}\right)=$ -1 . From the Frenet formulas, we also have $d \cdot \gamma^{\prime \prime}\left(s_{1}\right)=0, d \cdot \gamma^{(3)}\left(s_{1}\right)=\kappa\left(s_{1}\right)^{2}$, and $d \cdot \gamma^{(4)}\left(s_{1}\right)=0$. Thus,

$$
d \cdot \gamma(t)=d \cdot \gamma\left(s_{1}\right)+\left(s_{1}-t\right)+\kappa\left(s_{1}\right)^{2}\left(t-s_{1}\right)^{3} / 6+\mathcal{O}\left(\left(t-s_{1}\right)^{5}\right) .
$$

Similarly, expanding the cubic term in $Z_{1}(t)$ yields

$$
Z(t)^{3} / 3=\frac{1}{6} \kappa\left(s_{1}\right)^{2}\left(s_{1}-t\right)^{3}+\frac{1}{6} \kappa\left(s_{1}\right) \kappa^{\prime}\left(s_{1}\right)\left(s_{1}-t\right)^{4}+\mathcal{O}\left(\left(s_{1}-t\right)^{5}\right) .
$$

The difference between the latter two expressions, i.e., the phase of (3.13), is

$$
d \cdot \gamma\left(s_{1}\right)+\left(s_{1}-t\right)+\mathcal{O}\left(\left(t-s_{1}\right)^{4}\right) .
$$

Since $d \cdot \gamma\left(s_{1}\right)$ is independent of $t$, it can be absorbed into the constants of the asymptotic expressions. Thus, the phase of the MT expansion agrees with the phase of the GTD expression only up to third order near the shadow boundary. The exponential decay of both expansions, given by the $k^{1 / 3}$ term in the exponential, does agree perfectly. Any change in the function $Z(t)$ that corrects for the leading order term of the phase would necessarily cause a mismatch in the $k^{1 / 3}$ term. Thus, surprisingly enough, the predictions of GTD and the MT expansion differ in the shadow region by a phase factor. Only one of them can be accurate, and numerical experience indicates that the GTD expression is more appropriate to use.

3.3.4. An apparent contradiction. The phase difference between MT expansion and GTD does not seem to be widely recognized, although it is alluded to by Melrose and Taylor themselves in a following paper [26, p. 615]: “... Notice that the phase function $\psi_{1}$ is not really well-defined, since altering it by a term vanishing as $s^{3}$ at $B$ makes a change in $a_{1}$ which can be absorbed without altering the properties (1.36)-(1.39)."

The asymptotic behavior of the solution across the shadow boundary is accurately described by Babich and Buldyrev, following earlier work of Ludwig [24], in [2, Chapter 13]. However, the expressions involve a phase function $\xi(M, \gamma)$ that is not explicitly given but that can be expanded in a series near the shadow boundary $[2$, equation (13.2.1)]:

$$
\xi(M, \gamma) \sim \sum_{j=0}^{\infty} \xi_{j}(M) \gamma^{j} .
$$

Here, $M$ is a point in the field near the shadow boundary and $\gamma$ is a local coordinate with $\gamma=0$ at the shadow boundary: $M$ and $\gamma$ are ultimately identifiable with $t$ in (3.9) and the integration variable $z$ in (3.10), respectively. The function $\xi$ itself solves a problem-dependent eikonal-like equation; see also the description by Kim in [20, section 3.4]. Its expansion near the shadow boundary induces an approximation much like that of the approximate boundary condition (3.7). Kim shows in [20, section 3.4.1] how the MT expansion results from the description by Babich and Buldyrev, showing 


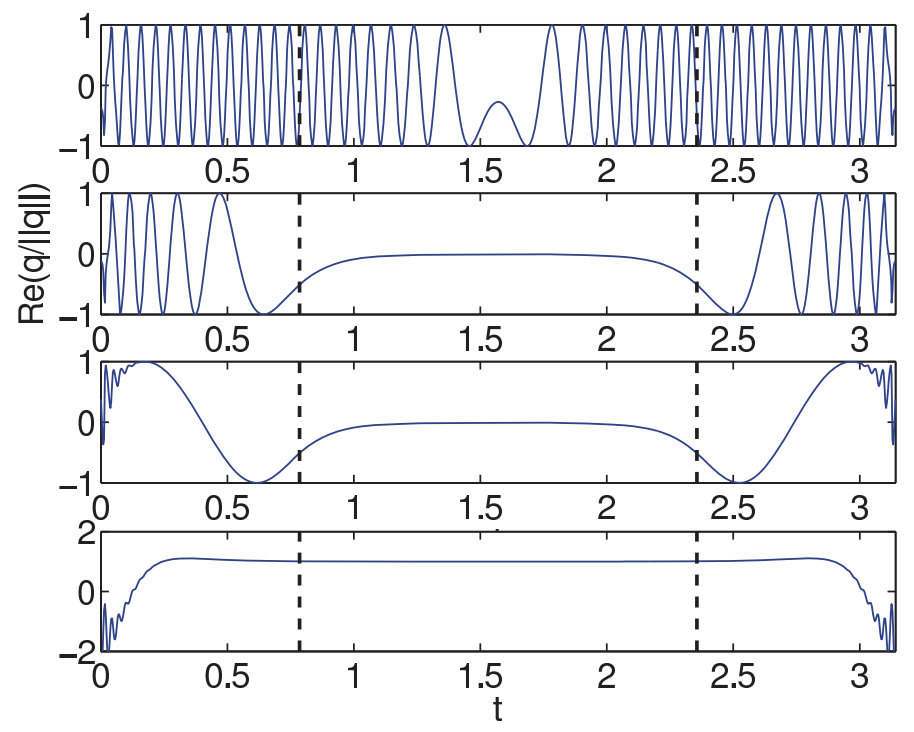

FIG. 4. The oscillations of the solution are visualized by showing the quantity $\operatorname{Re}(q /\|q\|)$. The solution is shown for the case of scattering by a circular object with radius $r=1 / 2$ and wavenumber $k=100$ (top), the result after extracting GO phase (second) and GTD phase (third). The bottom panel shows $\operatorname{Re}\left(q / \Phi^{M T}\right)$. The shadow boundaries are indicated with dashed lines.

explicitly that terms beyond third order are absorbed in the smooth functions $b_{l, m}(t)$ with the higher order terms, thus resulting in the phase discrepancy. ${ }^{1}$

Finally, it should be noted that there are no theoretical issues with the MT expansion. The main culprit is the fact that expansion (3.9) only guarantees that the difference between the left and the first terms of the right-hand side is algebraically small in $k$ in absolute size and not in relative size. Yet, in the shadow region, both quantities are exponentially small and their difference is small beyond all orders in $k$. It seems that the fourth order term of the phase becomes relevant only at a point where the solution is already exponentially small.

The advantages of the MT expansion remain: the geometric properties are decoupled from the complicated wavenumber dependency via the explicit functions $Z(t)$ and $\Psi(\tau)$. For the purpose of uniformly accurate phase extraction, it suffices to correct the phase of the MT expansion in the shadow region. Although not $C^{\infty}$ smooth, a simple phase correction can be devised by switching in the shadow region to the modified expression

$$
\Phi_{\text {mod }}^{M T}(t)=e^{i k\left(s_{1}-t+Z(\tau)^{3} / 3-d \cdot \gamma\left(s_{1}\right)\right)} \frac{n(t) \cdot d}{Z(t)} \Psi\left(k^{1 / 3} Z(t)\right), \quad t<s_{1} .
$$

The exponential in front of the definition essentially removes the phase of the MT expansion and adds in the phase of GTD. Note that both phases match to third order at the shadow boundary.

3.4. A comparison of different functions for phase extraction. Figure 4 shows the oscillations of the solution for a circle of radius $r=1 / 2$ with $k=100$. The

\footnotetext{
${ }^{1}$ The authors would like to thank Valery Smyshlaev for pointing out this connection and suggesting the reference [20].
} 


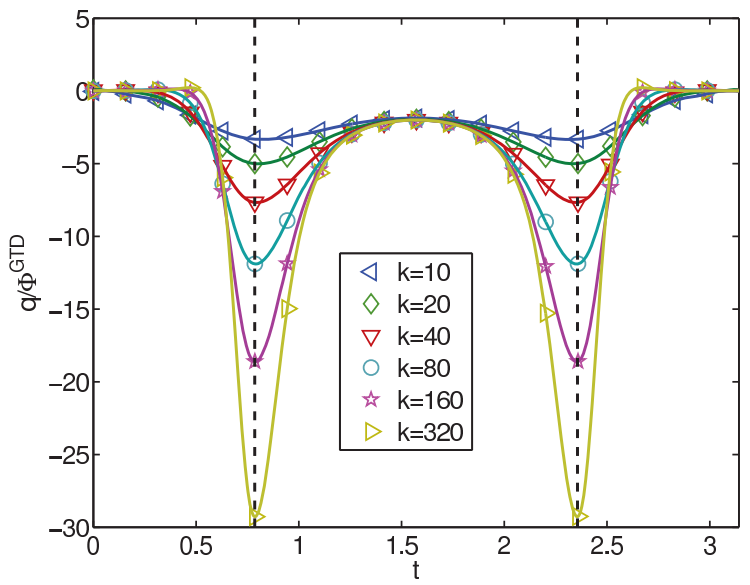

FIG. 5. The real part of the exact solution for the circle, with the same parameters as in Figure 4, after factoring out the globally defined GTD-phase. Wavenumber-dependent variations are visible near the shadow boundaries (vertical lines).

two lower panels here show the solution with the GO and GTD phases factored out. To be precise, the latter corresponds to using a single phase function that incorporates the dominating behavior of the GTD solution in the shadow behind both shadow boundaries,

$$
\Phi_{\text {global }}^{\text {GTD }}(t)= \begin{cases}\exp \left(i k\left(s_{1}-t-\alpha_{0} k^{1 / 3} \int_{s_{1}}^{t} \kappa^{2 / 3}(\tau) \mathrm{d} \tau\right)\right), & t<s_{1}, \\ \exp (i k d \cdot \gamma(t)), & s_{1}<t<s_{2}, \\ \exp \left(i k\left(t-s_{2}+\alpha_{0} k^{1 / 3} \int_{s_{2}}^{t} \kappa^{2 / 3}(\tau) \mathrm{d} \tau\right)\right), & t>s_{2} .\end{cases}
$$

After factoring out the GTD-phase, there remains an unknown function that is nonoscillatory, except in the deep shadow. Oscillations appear in the deep shadow due to the fact that the solution is a sum of two contributions - recall that two rays originate at each of the two shadow boundaries propagating in opposite directions. In the middle of the shadow region, these two contributions are of similar size, and the dominating behavior of either one of them is not sufficient for describing the total.

The GTD approximation is valid in illuminated and shadow regions but does show breakdown as $k \rightarrow \infty$ in the boundary between these regions. This is not very apparent in case of a circular scatterer and $k=100$ as shown in the bottom panel of Figure 4. Figure 5 shows the real part of the solution for the circle after extraction of the GTD-phase function. The remainder is indeed nonoscillatory almost everywhere, but exhibits large variations near the shadow boundary. The size of the deflection increases with $k$. This behavior can be seen as a boundary layer, which we would also like to extract from the solution as it introduces $k$-dependent variation in the solution which prevents a discretization with a fixed number of degrees of freedom. As this boundary layer does not really correspond to oscillations with a certain phase, one may think of the MT-phase function as being a "generalized phase function," extracting not just phases of oscillations but any wavenumber-dependent variation from the solution.

Finally, we illustrate the result after the extraction of the generalized, uniform phase functions (3.11) and (3.15) in Figure 6. The remainder is essentially one on large 

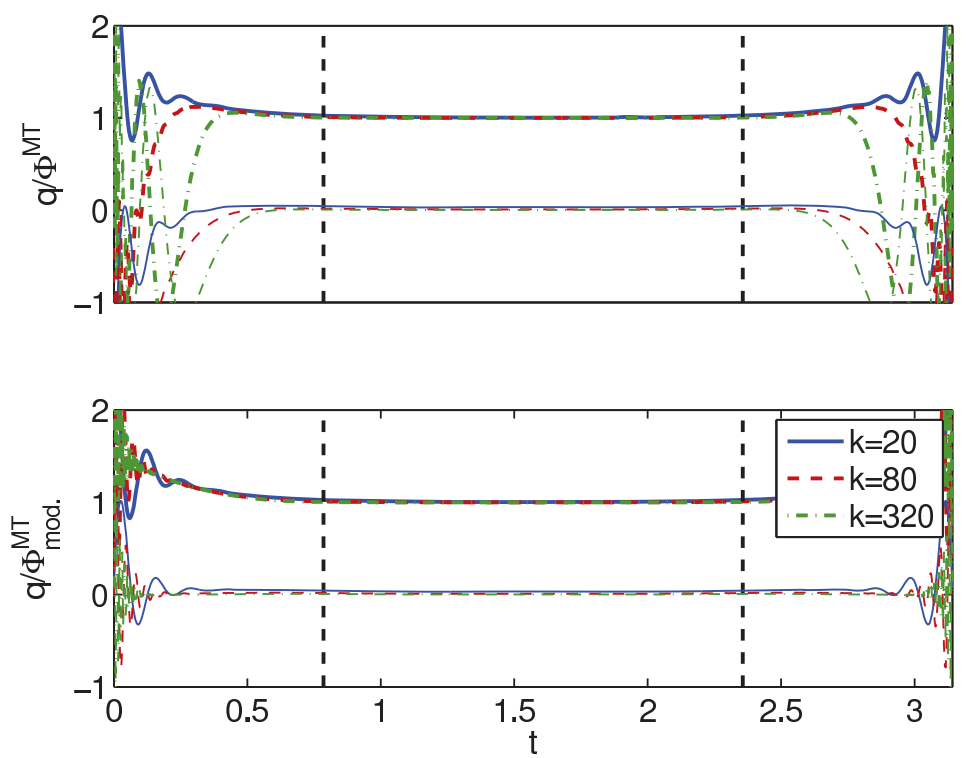

FIG. 6. Real and imaginary parts of the exact solution for the same circular scatterer as in Figure 4, after factoring out the uniformly accurate MT phase functions. In each of the two plots, the imaginary parts are below the real parts. Top: original form based on (3.11). Oscillations are still present in the shadow. Bottom: result after phase correction based on (3.15). Oscillations only appear in the deep shadow region where the creeping rays in opposite directions overlap.

parts of the scatterer. There is a similar breakdown of the solution as in the case of GTD in the deep shadow. In addition to having the same problem with overlapping solutions in the deep shadow, the first uniform phase function (3.11) also leads to oscillations in the shadow, since it is essentially local to the shadow boundary (top panel). The corrected phase function does not have this problem (bottom panel).

4. Two numerical methods. We devise two numerical methods based on extraction of the MT uniformy accurate phase functions, which are given by (3.11) or (3.15) near the first shadow boundary. To that end, we must formulate a globally valid phase function which agrees with the local expressions near each of the two shadow boundaries. We explore two ways of doing so:

1. In the first method, we construct a single function $Z(t)$ with simple zeros at $s_{1}$ and $s_{2}$, in complete accordance with the formulation of Theorem 1. This function is based on the expressions (3.14) for $Z_{1}(t)$ and $Z_{2}(t)$ near each of the shadow boundaries.

2. In the second method, we explicitly approximate the two contributions originating at each of the two shadow boundaries separately, in a way that is similar to the partition of unity finite element method (PUFEM) [25].

Both methods are defined in more detail below.

The results for both approaches are comparable. The first method is closer to the theory. The second method offers more flexibility and shows that the PUFEM formulation is well suited to combine knowledge from different asymptotic expansions valid in different parts of a problem. In particular, one could easily adapt the scheme to factor out all three phase functions $\Phi^{G O}, \Phi^{G T D}$, and $\Phi^{M T}$ in an overlapping way. We also attempt to adapt the scheme in order to track the individual creeping rays in the region where they overlap. 
Both methods rely on a partition of unity and a localization around each shadow boundary point. Thus, we define two intervals $\left[a_{1}, b_{1}\right]$ and $\left[a_{2}, b_{2}\right]$, both in $[0, L]$ and such that

$$
0<a_{1}<s_{1}<b_{1}<a_{2}<s_{2}<b_{2}<L .
$$

We define the partitioning functions as

$$
\xi_{1}(t)=\left\{\begin{array}{ll}
1, & t<b_{1}, \\
0, & t>a_{2},
\end{array} \quad \text { and } \quad \xi_{1}(t)+\xi_{2}(t)=1, \quad t \in \mathbb{R},\right.
$$

with, in addition, $\xi_{1}(t)>0, t \in\left(b_{1}, a_{2}\right)$. The computational domain is always intended to be the interval $\left[a_{1}, b_{2}\right]$.

4.1. Method one: A global function $Z$. In the first variant we define a globally valid function $Z$ from Eq. (3.14) using the partitioning functions $\xi_{1}$ and $\xi_{2}$ defined above:

$$
Z(t)=\xi_{1}(t) Z_{1}(t)+\xi_{2}(t) Z_{2}(t), \quad t \in[0, L] .
$$

In turn, this function defines a global and uniformly accurate phase function

$$
\Phi_{\text {global }}^{M T}(t):= \begin{cases}e^{i k\left(s_{1}-t+Z(t)^{3} / 3-d \cdot \gamma\left(s_{1}\right)\right) \frac{n(t) \cdot d}{Z(t)} \Psi\left(k^{1 / 3} Z(t)\right),} & 0<t<s_{1}, \\ e^{i k d \cdot \gamma(t) \frac{n(t) \cdot d}{Z(t)} \Psi\left(k^{1 / 3} Z(t)\right),} & s_{1}<t<s_{2}, \\ e^{i k\left(t-s_{2}+Z(t)^{3} / 3-d \cdot \gamma\left(s_{2}\right)\right)} \frac{n(t) \cdot d}{Z(t)} \Psi\left(k^{1 / 3} Z(t)\right), & s_{2}<t<L .\end{cases}
$$

The phase correction limits the smoothness of $\Phi^{M T}$ across the shadow boundaries. However, recall that the phases to the left and to the right of the shadow boundaries do agree to third order. Hence, this is not a limitation in practice, as we intend to use low order basis functions in the discretization.

Next, we define a spline space $V_{N}\left[a_{1}, b_{2}\right]$, spanned by $N$ splines $\left\{\phi_{j}\right\}_{j=1}^{N}$ of low degree $l=1$ or $l=3$. We solve integral equation (2.7) approximately, using a collocation approach with collocation points $\left\{t_{i}\right\}_{i=1}^{N}$ that coincide with the nodes of the splines. This leads to an approximation $q_{s} \approx \varphi^{N} \in V_{N}\left[a_{1}, b_{2}\right]$.

Note that the solution is not defined in the deep shadow, i.e., for $\tau<a_{1}$ and $\tau>b_{2}$. As we have seen above, the solution in the deep shadow does not allow an ansatz with a single phase function due to the overlap of creeping rays. A common approach to truncate the computational domain in the deep shadow is to approximate the solution by zero, since it is exponentially small. However, in the proposed representation of the solution, truncating the smooth solution $\varphi_{N}$ in the shadow leads to inaccuracies, since $q_{s}$ is not expected to be small in the deep shadow. Indeed, the exponential decay is extracted in the phase function and we have $q_{s} \approx 1$ at the truncation point. Instead, we continue $\varphi_{N}$ into the deep shadow using its value at the truncation points, i.e., using $\varphi_{N}\left(a_{1}\right)$ and $\varphi_{N}\left(b_{2}\right)$, respectively. This choice ensures, by the theory of Filon-type quadrature, a suitable approximation to the oscillatory integral in the left-hand side of (2.7) [17]. Thus, we modify the solution ansatz as follows:

$$
q(t) \approx \begin{cases}\varphi_{N}\left(a_{1}\right) \Phi_{\text {global }}^{M T}(t), & t<a_{1} \\ \varphi_{N}(t) \Phi_{\text {global }}^{M T}(t), & a_{1} \leq t \leq b_{2} \\ \varphi_{N}\left(b_{2}\right) \Phi_{\text {global }}^{M T}(t), & t>b_{2}\end{cases}
$$


The collocation approach can now be formulated as

$$
\sum_{j=1}^{N} c_{j}\left[\alpha_{i, j}+w_{i}^{1} \phi_{j}(a)+w_{i}^{2} \phi_{j}(b)\right]=-e^{i k d \cdot \gamma\left(t_{i}\right)}, \quad i=1,2, \ldots, N
$$

where

$$
\begin{aligned}
\alpha_{i, j} & =\int_{0}^{L} K\left(\tau, t_{i}\right) \Phi_{\text {global }}^{M T}(\tau) \phi_{j}(\tau) \mathrm{d} \tau, \\
w_{i}^{1} & =\int_{0}^{a_{1}} K\left(\tau, t_{i}\right) \Phi_{\text {global }}^{M T}(\tau) \mathrm{d} \tau \\
w_{i}^{2} & =\int_{b_{2}}^{L} K\left(\tau, t_{i}\right) \Phi_{\text {global }}^{M T}(\tau) \mathrm{d} \tau .
\end{aligned}
$$

This linear system has moderate size, and a direct solver can be applied.

4.2. Method two: Using two local $Z$-functions. The global function $Z$ defined in the first method is somewhat arbitrary in the illuminated region. In the second method, we aim for a discretization that mimicks the structure of the different asymptotic regimes explicitly, which is similar to the general PUFEM. The two $Z$ functions corresponding to the shadow boundaries are not connected.

We write an ansatz for the solution of the form

$$
q(t)=q_{s, 1}(t) \Phi_{M T, 1}(t)+q_{s, 2}(t) \Phi_{M T, 2}(t),
$$

where the two phase functions are defined as

$$
\begin{aligned}
& \Phi_{M T, 1}(t)=\Phi^{M T}\left(s_{1}-t\right) \xi_{1}(t), \\
& \Phi_{M T, 2}(t)=\Phi^{M T}\left(t-s_{2}\right) \xi_{2}(t) .
\end{aligned}
$$

Note that this ansatz ensures that the unknowns $q_{s, 1}$ and $q_{s, 2}$ are both $\mathcal{O}(1)$ except in the deep shadow. Note also that these phase functions are defined for $t<0$ and $t>L$. This property will be used later on in the next section.

We suggest a spline collocation method for $q_{s, 1}$ and $q_{s, 2}$, using two natural spline spaces, $V_{N}^{1}\left[a_{1}, a_{2}\right]$ and $V_{N}^{2}\left[b_{1}, b_{2}\right]$, spanned by the spline basis functions $\left\{\phi_{j}^{1}\right\}_{j=1}^{N}$ and $\left\{\phi_{j}^{2}\right\}_{j=1}^{N}$, respectively. As before, we extend the solution ansatz into the deep shadow by its values at the truncation points,

$$
q(t) \approx \begin{cases}\varphi_{N, 1}\left(a_{1}\right) \Phi_{M T, 1}(t), & t<a_{1} \\ \varphi_{N, 1}(t) \Phi_{M T, 1}(t)+\varphi_{N, 2}(t) \Phi_{M T, 2}(t), & a_{1} \leq t \leq b_{2} \\ \varphi_{N, 2}\left(b_{2}\right) \Phi_{M T, 2}(t), & t>b_{2}\end{cases}
$$

The collocation method for (2.5) is then obtained using a set of collocation points $\left\{t_{i}\right\}_{i=1}^{2 N}$ that is the union of the sets of spline nodes associated with $V_{N}^{1}$ and $V_{N}^{2}$, and enforcing the equation at these points:

$$
\begin{aligned}
\sum_{j=1}^{N}\left[c_{1, j}\left(w_{i}^{1} \phi_{j}^{1}\left(a_{1}\right)+\alpha_{i, j}^{1}\right)+c_{2, j}\left(w_{i}^{2} \phi_{j}^{2}\left(b_{2}\right)\right.\right. & \left.\left.+\alpha_{i, j}^{2}\right)\right] \\
& =-e^{i k d \cdot \gamma\left(t_{i}\right)}, \quad i=1,2, \ldots, 2 N,
\end{aligned}
$$


where

$$
\begin{aligned}
\alpha_{i, j}^{1} & =\int_{0}^{L} K\left(\tau, t_{i}\right) \Phi_{M T, 1}(\tau) \phi_{j}^{1}(\tau) \mathrm{d} \tau \\
\alpha_{i, j}^{2} & =\int_{0}^{L} K\left(\tau, t_{i}\right) \Phi_{M T, 2}(\tau) \phi_{j}^{2}(\tau) \mathrm{d} \tau \\
w_{i}^{1} & =\int_{0}^{a_{1}} K\left(\tau, t_{i}\right) \Phi_{M T, 1}(\tau) \mathrm{d} \tau, \quad w_{i}^{2}=\int_{b_{2}}^{L} K\left(\tau, t_{i}\right) \Phi_{M T, 2}(\tau) \mathrm{d} \tau .
\end{aligned}
$$

4.3. Additional treatment of the deep shadow behavior. The problem associated with overlapping contributions in the deep shadow implies that the proposed numerical methods still disregard an exponentially small contribution. For any fixed value of the wavenumber, this exponentially small contribution can still be significant. One way to proceed would be to extend the two spline spaces of method two such that they wrap around in the deep shadow and overlap with each other. This approach presents serious numerical difficulties, which are not further pursued in this paper.

However, a simple modification to the second scheme does account to some extent for the overlapping rays. Since the approximate solutions are already extended by a constant outside of the definition of the splines, one can let the two rays wrap around by extending this constant even further beyond the deepest shadow point. More specifically, we modify the ansatz (4.3) to be

$$
q(t) \approx \begin{cases}\varphi_{N, 1}\left(a_{1}\right) \Phi_{M T, 1}(t)+\varphi_{N, 2}\left(b_{2}\right) \Phi_{M T, 2}(L+t), & t<a_{1}, \\ \varphi_{N, 1}(t) \Phi_{M T, 1}(t)+\varphi_{N, 2}(t) \Phi_{M T, 2}(t) & \\ \quad+\varphi_{N, 1}\left(a_{1}\right) \Phi_{M T, 1}(t-L)+\varphi_{N, 2}\left(b_{2}\right) \Phi_{M T, 2}(L+t), & a_{1} \leq t \leq b_{2}, \\ \varphi_{N, 2}\left(b_{2}\right) \Phi_{M T, 2}(t)+\varphi_{N, 1}\left(a_{1}\right) \Phi_{M T, 1}(t-L), & t>b_{2} .\end{cases}
$$

Subsequently, the only required modification to the method is to redefine the integrals $w_{i}^{1}$ and $w_{i}^{2}$ to be

$$
\begin{aligned}
& \tilde{w}_{i}^{1}=\int_{a_{1}-L}^{a_{1}} K\left(\tau, t_{i}\right) \Phi_{M T, 1}(\tau) \mathrm{d} \tau \\
& \tilde{w}_{i}^{2}=\int_{b_{2}}^{b_{2}+L} K\left(\tau, t_{i}\right) \Phi_{M T, 2}(\tau) \mathrm{d} \tau .
\end{aligned}
$$

5. Numerical results. We illustrate the accuracy of both methods with a number of examples. We focus on the accuracy that can be gained compared to factoring out only the phase in the illuminated region, i.e., we mainly illustrate the improved accuracy across the shadow boundary that results from factoring out the adverse wavenumber-dependent behavior in the transitional regions and in the shadow. In these experiments, the integrals corresponding to matrix entries are resolved with a generic numerical quadrature routine, the quadgk-function in MATLAB. This routine handles the singular integrals in a straightforward manner. All the integrals are resolved with a tolerance of $10^{-11}$. The special function $\Psi$ was precomputed to machine precision at a large number of Chebyshev points on the interval $[-10,7]$ using the steepest descent method [14]. The function $\Psi$ can accordingly be evaluated efficiently and to high precision at any point in this interval through its Chebyshev expansion. 


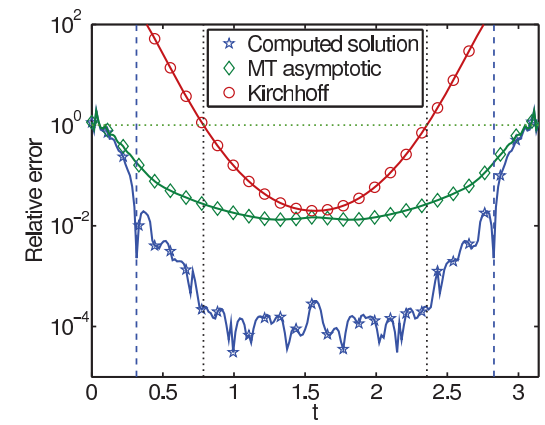

(a) Without phase correction

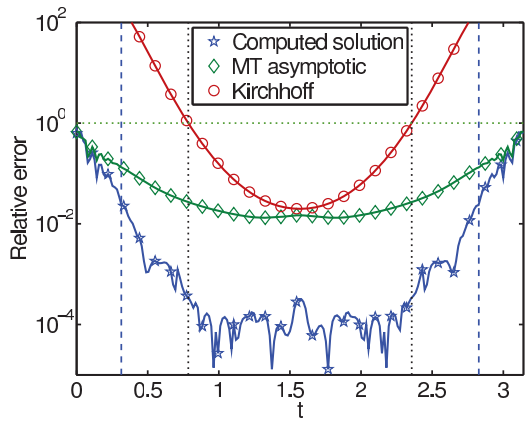

(b) With phase correction

FIG. 7. Illustration of the relative error of the approximation for $q$ in the first method, for scattering by a circular object with $k=50$ and using $N=20$ linear spline basis functions. $A$ global phase function is extracted with and without phase correction. The spline space extends from $a_{1}=L / 10$ to $b_{2}=9 L / 10$ (dashed lines). Gray lines correspond to shadow boundaries.

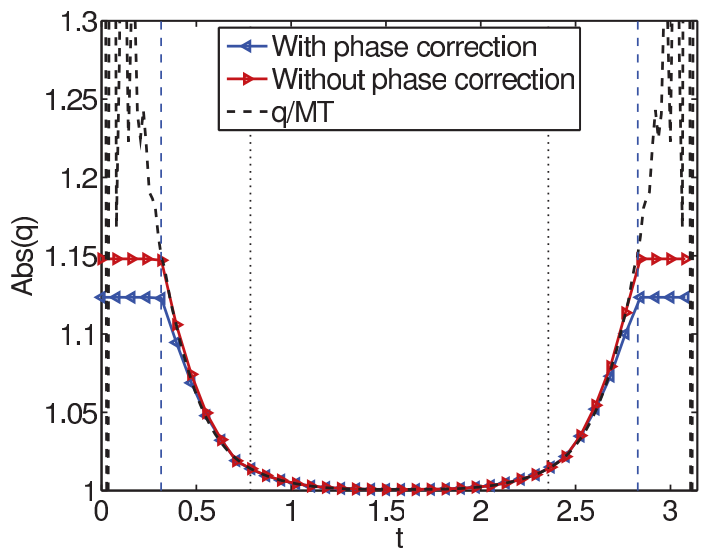

FIG. 8. The absolute value of the computed approximations to $q_{s}$ corresponding to Figure 7 , with and without phase correction in the shadow regions. The approximation is extended by a constant outside the support of the spline space. The dashed line shows the exact solution $q$ divided by the corrected MT asymptotics.

5.1. A circular scatterer. First, the two numerical methods are applied to a circular scatterer, for which an analytic solution is available in the form of an infinite series involving Bessel functions.

The first method computes the solution with nearly four digits of accuracy in the illuminated region with a very modest number of unknowns, $N=20$, and with $k=50$; see Figure 7. The relative accuracy is clearly uniform across the shadow boundaries. The global phase function is used without phase correction in the left panel and with phase correction in the right panel: the improvement in the shadow is clearly visible. It should also be noted that a relative accuracy between $1 \%$ and $10 \%$ is maintained even beyond the truncation points of the spline space. This is due to extending the approximate solution by a constant, which multiplies the exponentially decaying phase function. This approximation remains accurate for a little while, until the effect of the overlapping creeping rays limits the relative acccuracy in the deeper shadow. The corresponding approximations to the smooth function $q_{s}$ are shown in Figure 8. 


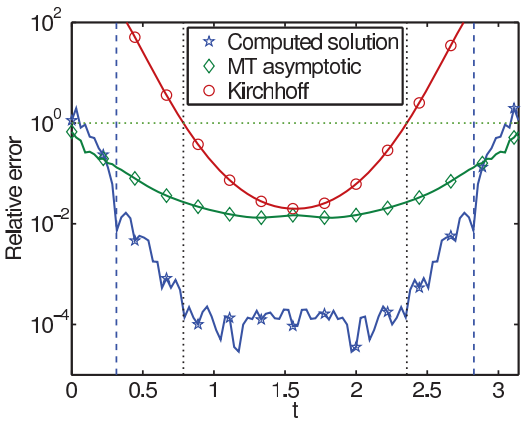

(a) Without phase correction

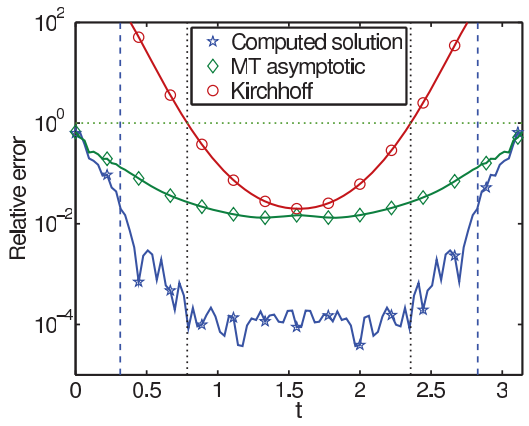

(b) With phase correction

FIG. 9. Relative errors in the approximation of $q$ computed by the second method for the same problem as in Figure 7 , using $N=10$ hat functions in each spline space.

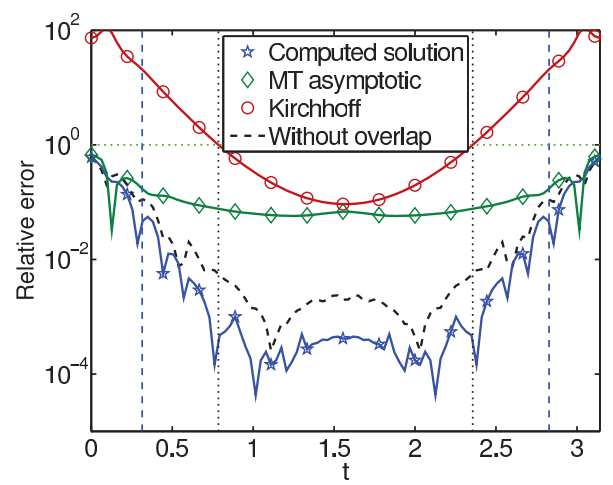

FIG. 10. Method 2 implemented with overlapping creeping rays using $k=10$ and $N=10$.

Also shown in Figure 7 are the Kirchhoff approximation and the leading order term of the MT expansion. The Kirchhoff approximation breaks down near the shadow boundaries; the MT expansion is clearly uniform. However, the improvement from the numerical approach is very significant: the accuracy improves by two orders of magnitude, even well beyond the shadow boundaries.

The results of the second method for the same problem are shown in Figure 9. The accuracy is comparable to the results of the first method, using the same total number of degrees of freedom. The accuracy can be improved further by implementing the overlapping creeping rays in the deep shadow as described in section 4.3. The effect will be more noticeable at lower frequencies. For Figure 10 we redid the experiment, but this time with $k=10$, and then compared the method implemented with and without overlapping creeping rays. A clear improvement can be observed.

In order to show robustness with respect to increasing $k$, the experiment is repeated for varying $k$, using the second method with overlapping creeping rays. The results in Figure 11 show that there is no qualitative difference in the error behavior for increasing $k$.

It should be noted that achieving small relative error on an exponentially decaying solution is extremely difficult. The absolute errors of the approximations of both methods are shown in Figure 12. All methods achieve a uniformly small absolute error 


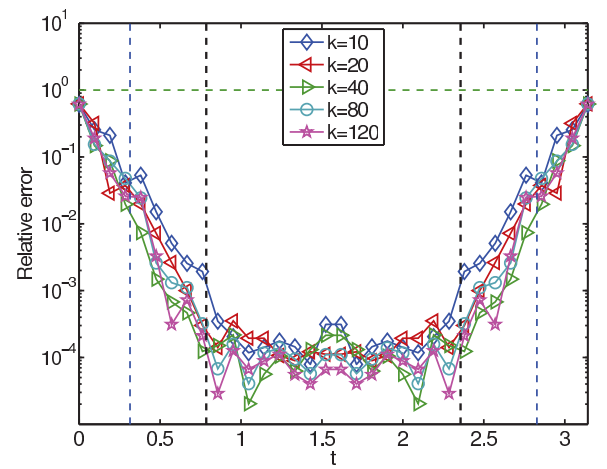

FIG. 11. Same experiment as in Figure 9(b) but for increasing values of $k: k=20,40,80,120$.

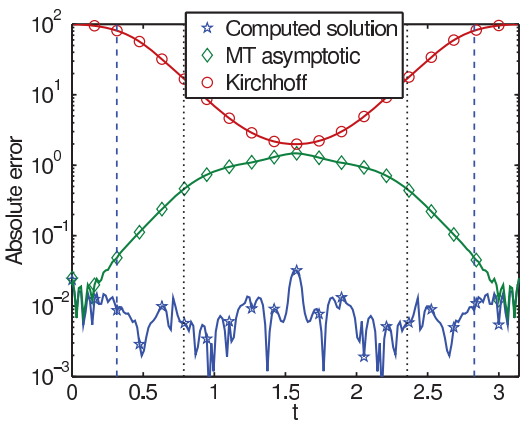

(a) Method one

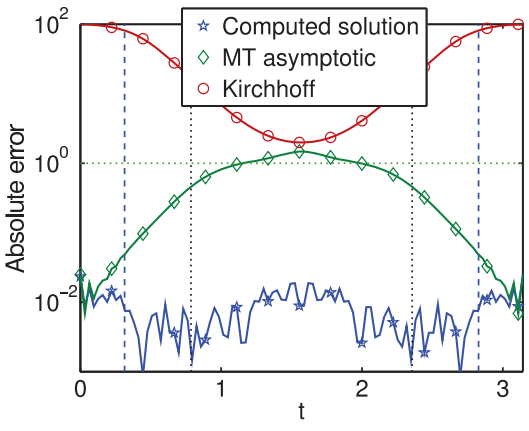

(b) Method two

FIG. 12. Absolute errors of the approximation of $q$ using method one and method two (with overlap), corresponding to the relative errors shown in Figure 7 and Figure 9(b).

throughout the computational domain. This explains the lack of relative accuracy in the deepest shadow: relative accuracy cannot be achieved if the size of the solution is comparable to the overall absolute error in the computations.

Finally, note that the absolute error is defined here as

$$
\left|q-\varphi_{N} \Phi_{\text {global }}^{M T}\right|
$$

It is common in literature to assess the absolute error using

$$
\frac{\left|q-\varphi_{N} \Phi_{\text {global }}^{M T}\right|}{k}
$$

instead. The division by the wavenumber is motivated by the fact that the solution is $\mathcal{O}(k)$ in the illuminated region and dividing by $k$ results in a normalization. However, the solution is no longer $\mathcal{O}(k)$ near the shadow boundary, and, hence, dividing by $k$ results in an underestimation of the actual absolute error of the solution.

5.2. An ellipse-shaped scatterer. An analytic solution is no longer available for an ellipse-shaped scatterer. Moreover, both the parametrization and the $Z$-function are not easily expressed in closed form. By considering an ellipse that is slightly skew relative to the incident wave, the problem is nonsymmetric as well. 


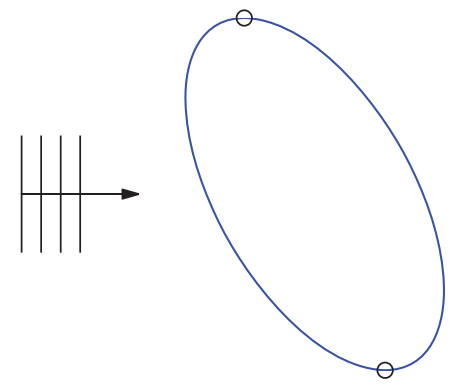

(a)

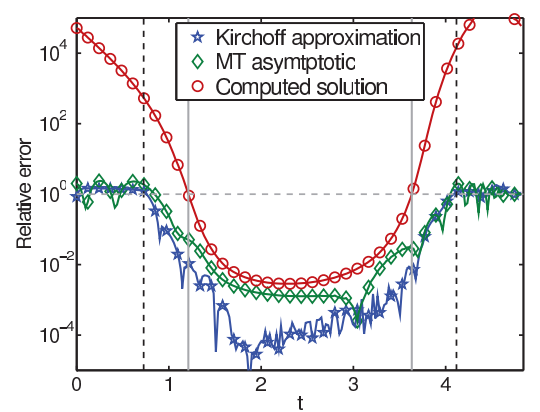

(b)

FIG. 13. Relative error of the approximation of $q$ using the second method with phase correction for an ellipse-shaped scatterer, with $k=50$, using $N=15$ hat functions in each spline space and truncating at $a_{1}=0.15 \mathrm{~L}$ and $b_{2}=0.85 \mathrm{~L}$.

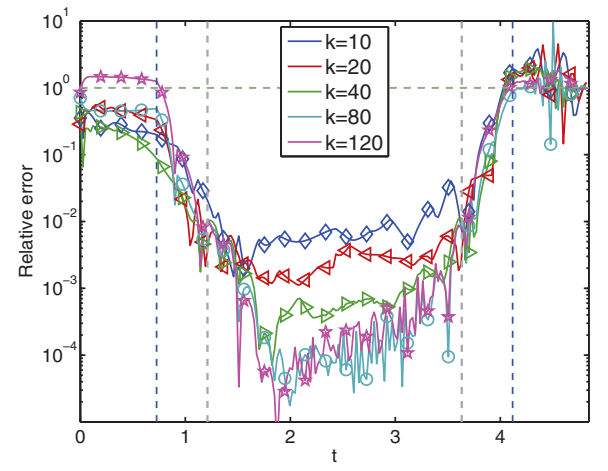

FIG. 14. Same experiment as in Figure 13(b) but for increasing values of $k: k=10,20,40,80,120$.

For this experiment, the parametrization was constructed using a Fourier-based representation of the scatterer, obtained by interpolating the ellipse at 100 points. The function $Z$ was approximated using Gaussian quadrature. A reference solution to the problem was obtained using a Galerkin BEM method [16] with 4096 unknowns. The ellipse used here has a minor semiaxis of length $1 / 2$ and a major semi-axis of length 1 , rotated $30^{\circ}$ relative to the $y$-axis. The incident plane wave travels in the direction of the positive $x$-axis, as illustrated in Figure 13(a).

The numerical experiment was carried out using the second method with $N=15$ hat functions in each spline space. One observes a similar gain in accuracy by using the numerical method as before, when compared to the Kirchhoff approximation and the MT expansion. However, it seems that the second shadow boundary poses more problems than the first one. This is probably due to a more rapid change in the curvature behind this shadow boundary.

The experiment that produced Figure 11 was repeated for the case of an ellipseshaped scatterer. The results in Figure 14 show that also in this case the error is bounded as $k$ increases. Note that in this case the error actually diminishes with increasing $k$.

Acknowledgments. The authors wish to thank Valery P. Smyshlyaev for his assistance in preparing this manuscript and Dave Hewett for his helpful comments. 


\section{REFERENCES}

[1] A. Asheim And D. Huybrechs, Local solutions to high-frequency $2 D$ scattering problems, J. Comput. Phys., 229 (2010), pp. 5357-5372.

[2] V. M. Babich and V. S. Buldyrev, Short-Wavelength Diffraction Theory, Springer-Verlag, Berlin, 1991.

[3] O. Bruno and C. Geuzaine, An O(1) integration scheme for three-dimensional surface scattering problems, J. Comput. Appl. Math., 204 (2007), pp. 463-476.

[4] O. P. Bruno, C. A. Geuzaine, J. A. Monro, Jr., And F. Reitich, Prescribed error tolerances within fixed computational times for scattering problems of arbitrarily high frequency: The convex case, Philos. Trans. R. Soc. A, 362 (2004), pp. 629-645.

[5] V. S. Buslaev, Short-wave asymptotic behaviour in the problem of diffraction by smooth convex contours, Boundary Value Problems of Mathematical Physics, Part 2, Vol. 73, Nauka, Moscow, 1964, pp. 14-117.

[6] S. N. Chandler-Wilde, I. G. Graham, S. Langdon, and E. A. Spence, Numericalasymptotic boundary integral methods in high-frequency acoustic scattering, Acta Numer., 21 (2012), pp. 89-305.

[7] D. Colton And R. Kress, Integral Equation Methods in Scattering Theory, Wiley, New York, 1983.

[8] V. Domínguez, I. G. Graham, and V. P. Smyshlyaev, A hybrid numerical-asymptotic boundary integral method for high-frequency acoustic scattering, Numer. Math., 106 (2007), pp. 471-510.

[9] V. Domínguez, I. G. Graham, and V. P. Smyshlyaev, Stability and error estimates for Filon-Clenshaw-Curtis rules for highly oscillatory integrals, IMA J. Numer. Anal., 31 (2011), pp. 1253-1280.

[10] V. A. Fock, Electromagnetic Diffraction and Propagation Problems, Pergamon Press, New York, 1965.

[11] M. Ganesh and S. C. Hawkins, A fully discrete Galerkin method for high frequency exterior acoustic scattering in three dimensions, J. Comput. Phys., 230 (2011), pp. 104-125.

[12] E. GiLadi, Asymptotically derived boundary elements for the Helmholtz equation in high frequencies, J. Comput. Appl. Math., 198 (2007), pp. 52-74.

[13] D. Huybrechs and S. Olver, Highly oscillatory quadrature, in Highly Oscillatory Problems: Computation, Theory and Application, B. Engquist, A. Fokas, E. Hairer, and A. Iserles, eds., Cambridge University Press, Cambridge, 2009, pp. 25-50.

[14] D. Huybrechs and S. Vandewalle, On the evaluation of highly oscillatory integrals by analytic continuation, SIAM J. Numer. Anal., 44 (2006), pp. 1026-1048.

[15] D. Huybrechs AND S. VANDEWAlle, A sparse discretisation for integral equation formulations of high frequency scattering problems, SIAM J. Sci. Comput., 29 (2007), pp. 2305-2328.

[16] D. HuYBrechS AND S. VANDEWALLE, An efficient implementation of boundary element methods for computationally expensive Green's functions, Eng. Anal. Bound. Elem., 32 (2008), pp. 621-632.

[17] A. Iserles AND S. P. NøRSETt, Efficient quadrature of highly oscillatory integrals using derivatives, Proc. R. Soc. Lond. A, 461 (2005), pp. 1383-1399.

[18] J. B. KeLLER, Diffraction of a convex cylinder, IRE Transactions on Antennas and Propagation, 4 (1956), pp. 312-321.

[19] J. B. Keller, Geometrical theory of diffraction, J. Opt. Soc. Amer., 52 (1962), pp. 116-130.

[20] T. KIm, Asymptotic and Numerical Methods for High-Frequency Scattering Problems, Ph.D. thesis, University of Bath, Bath, UK, 2012.

[21] R. G. Kouyoumuian and P. H. Pathak, A uniform geometrical theory of diffraction for an edge in a perfectly conducting surface, Proc. IEEE, 62 (1974), pp. 1448-1461.

[22] M. A. Leontovich And V. A. Fock, Solution of the problem of propagation of electromagnetic waves along the earth's surface by the method of parabolic equation, Acad. Sci. USSR. J. Phys, 10 (1946), pp. 13-24.

[23] D. Ludwig, Uniform asymptotic expansions at a caustic, Comm. Pure Appl. Math., 19 (1966), pp. 215-250.

[24] D. LuDwig, Uniform asymptotic expansion of the field scattered by a convex object at high frequencies, Comm. Pure Appl. Math., 20 (1967), pp. 103-138.

[25] J. M. Melenk and I. BabušKa, The partition of unity finite element method: basic theory and applications, Comput. Methods Appl. Math., 139 (1996), pp. 289-314.

[26] R. B. MeLrose, The radiation pattern of a diffracted wave near the shadow boundary, Comm. Partial Differential Equations, 11 (1986), pp. 599-672. 
[27] R. B. Melrose And M. E. TAYlor, Near peak scattering and the corrected Kirchhoff approximation for a convex obstacle, Adv. Math., 55 (1985), pp. 242-315.

[28] C. S. Morawetz And D. Ludwig, An inequality for the reduced wave operator and the justification of geometrical optics, Comm. Pure Appl. Math., 21 (1968), pp. 187-203.

[29] J.-C. NÉdÉLEC, Acoustic and Electromagnetic Equations, Appl. Math. Sci. 144, Springer, Berlin, 2001.

[30] R. H. Tew, S. J. Chapman, J. R. King, and J. R. Ockendon, Scalar wave diffraction by tangent rays, Wave Motion, 32 (2000), pp. 363-380. 